\title{
La aeronáutica como sector estratégico en España. Situación actual y perspectivas en Galicia
}

\author{
Ángel Miramontes Carballada (a) \\ (a) Departamento de Xeografía / Universidade de Santiago de Compostela; angel.miramontes@usc.es
}

\section{Resumen}

\begin{abstract}
La aeronáutica es un sector que, entre otras características, destaca porque reinvierte un porcentaje mayor en I+D+i que otras actividades, provocando un efecto positivo directo en la productividad, competitividad y ocasiona un efecto tractor en otras industrias. Estos son algunos de los hechos que han motivado que la industria aeronáutica lleve unas décadas siendo definida por algunos países, como estratégica para su desarrollo socioeconómico. Entre los que se encuentra España, donde desde el Gobierno español se han redactado y puesto en acción diferentes planes tecnológicos y estratégicos centrados en el desarrollo y apoyo al sector. Aunque con un grado de ejecución y resultados diversos. Además se mostrará la realidad de un territorio concreto como Galicia donde, entre otras actuaciones, se ha creado una red de agentes con el objetivo principal de dinamizar este sector, como el Centro de Innovación Aeroespacial de Galicia, el Consocio Aeronáutico Gallego, la colaboración de la Universidade de Vigo (desde la Escuela de Ingeniería de Telecomunicaciones y la Escuela de Ingeniería Aeronáutica y del Espacio), el Consorcio de la Zona Franca de Vigo o la Xunta de Galicia
\end{abstract}

Palabras claves: Aeronáutica, Sector Estratégico, España, Galicia.

\section{INTRODUCCIÓN}

El presente trabajo sigue una de las líneas clásicas dentro de la Geografía Económica, estudio de la influencia que tiene una determinada actividad económica sobre el territorio. Además de comprobar la evolución y significación de las variables económicas más generales como facturación o empleo, se trata de conocer los procesos de innovación que se generan, si existe una red de agentes o cual es el grado de relación entre los diferentes entes y/o organismos vinculados con esta actividad. Pues con el análisis de estas redes podremos conocer la importancia y futuro próximo que puede llegar a tener, en este caso, el sector aeronáutico en Galicia.

Para alcanzar este objetivo principal, dividimos este trabajo en una introducción centrada en revisar las características generales de los procesos de innovación. Un segundo apartado a modo de "radiografía" del sector aeronáutico en España, para concluir con un estudio de este sector en un espacio concreto como es Galicia. Eso sí, prestándole mayor atención al estudio de la presencia o ausencia de agentes públicos, privados o empresas de este sector y el grado de relación que mantienen entre ellos. Para finalizar con una breve reflexión a modo de conclusión.

Gracias a la amplia relación de trabajos realizados desde el grupo de Geografía Económica de la AGE (Asociación de Geógrafos Españoles), existe un cierto consenso sobre el término de innovación (Méndez, R., 2000, 2006; Méndez, R. y Alonso, J.L. 2000, 2002; Caravaca, I. et al, 2002; Alonso, Mª. P. y Miramontes, Á., 2003; Salom, J. 2003, Alonso, J. L. et al, 2004; Salom, J. y Albertos, J. M. 2009, 2013; Miramontes, Á. y Alonso, M‥ P., 2015). La innovación es un fenómeno social y económico que puede iniciarse desde diferentes ámbitos: por el esfuerzo individual o de un conjunto de actores vinculados con una actividad económica, las características naturales de un espacio, la presencia de un saber hacer fabril o la realización de una estrategia guiada desde la administración pública. Sin embargo, el protagonista y que permite el desarrollo de los procesos de innovación en un territorio son las empresas y, en mayor medida, la capacidad que tengan de conformar una red en la que interactúen los actores públicos, privados, asociaciones, etc. relacionados con esa actividad económica (Aydalot, P., 1986; Camagni, R., 1991, 2003; Maillat, D. 1995; Vázquez, A., 1999; Sforzi, F., 1999).

Por lo que es importante tener claro que la innovación es una actividad colectiva, que proviene de la acción de un conjunto de actores que establecen un sistema de relaciones basadas en la cooperación, pudiendo llegar a configurar una red de innovación. En donde la política de innovación se interpreta de manera genérica como un 
conjunto de acciones cuyo objetivo es incrementar la cantidad y la eficiencia de actividades innovadoras relativas a la creación, adaptación y adopción de nuevos o mejorados productos, procesos o servicios. Ello implica un elevado y heterogéneo número de actores, instrumentos y medidas (Smits et al., 2010; Borrás y Edquist, C., 2013).

Antes de trabajar y conocer las características estadísticas de un sector como es el aeronáutico, es fundamental incidir en que tiene una serie de peculiaridades que se repiten independientemente del territorio en el que se localice y se resumen en tres. En primer lugar se trata de una industria que requiere la existencia de apoyo por parte de las instituciones públicas, entre otros motivos por la necesidad de emplear enormes cantidades de recursos económicos. En segundo lugar, deben existir unas empresas tractoras que centralicen la actividad y, en tercer lugar la industria aeronáutica está sometida a una altísima exigencia de calidad y empleo altamente cualificado. Por estos motivos se incluye a la industria aeroespacial en uno de los pilares de la economía de alto valor añadido a escala mundial.

Según los datos del INE y TEDAE (Asociación Española de Empresas Tecnológicas de Defensa, Aeronáutica y Espacio), la industria aeronáutica española ha triplicado su tamaño en los últimos 15 años. En la actualidad es una actividad con un negocio de cerca de 8.000 millones de euros y más de 100.000 empleos (TEDAE, 2017). Sin embargo, desde el sector español están palpando como en el mercado global cada vez hay más actores y como desde la propia UE, se ha visto a la industria aeronáutica como la clase de industria de alta tecnología y alto valor añadido que no puede ser exportada. A lo que hay que añadir que desde la TEDAE afirman que España es uno de los diez países del mundo capaz de poner en vuelo un avión (TEDAE, 2015).

Todas estas son las motivaciones que justifican la necesidad de comenzar a profundizar en el estudio de este sector en España y, en mayor medida en Galicia que es un territorio localizado en el noroeste de la Península Ibérica y periferia dentro del Sur de Europa, al que se le puede presuponer que no dispone de las características de partida ideales para el desarrollo de una actividad industrial de estas características. De hecho en Galicia, si existe una considerable actividad industrial. Aunque esta industria está focalizada en unas determinadas actividades fabriles tradicionales como la industria textil y moda, la producción y transformación de madera o la pesca. Además estas actividades se caracterizan por concentrar los mayores volúmenes de empleo y facturación en una o dos empresas dentro de cada sector como son los casos de INDITEX, FINSA, S.A. o ENCE. Por lo que aumenta el interés en conocer, cómo se comporta un sector como el aeronáutico y las consecuencias de la red de agentes que existen a día de hoy en torno a su actividad, en un territorio con predominio de actividades industriales tradicionales.

\section{CARACTERÍSTICAS PRINCIPALES DEL SECTOR AERONÁUTICO. EL CASO DE ESPAÑA}

Como ya queda reflejado en el título de este trabajo, tanto de la UE como desde España a la industria aeronáutica se le atribuye un papel estratégico. Sin entrar en más detalle, la Comisión Europea ha integrado la Política Aeroespacial Europea en el núcleo del crecimiento inteligente sostenible e inclusivo al que se refiere la estrategia "Europa 2020".

En el ámbito español, el Plan Integral de Política Industrial 2020 (PIN 2020), elaborado por el Ministerio de Industria, reconoce al sector aeroespacial como uno de los sectores estratégicos futuros para la industria española, habida cuenta de su mayor potencial de crecimiento, su mayor capacidad de arrastre sobre la industria y su orientación a la exportación.

Como adelantamos en la introducción, desde un punto de vista cualitativo este sector presenta una serie de aspectos que le confieren una identidad peculiar y diferenciada del resto de sectores productivos, entre los que se pueden mencionar los siguientes (TEDAE, 2015):

- El mercado aeronáutico tiene un doble componente civil y militar de forma que la demanda en ambos mercados puede oscilar de manera diferente. Además la parte militar tan sólo tiene como clientes a la administración (nacional y/o internacional).

- El sector tiene una estructura empresarial liderada por una serie de grupos industriales, sustentados en una red de grandes, medianas y pequeñas empresas subcontratadas, con una alta dependencia entre ellos.

Por otro lado, su carácter estratégico viene explicado por factores como los siguientes:

- El componente militar de la industria aeronáutica le confiere un carácter clave para la seguridad y defensa nacional. 
- Es un sector generador de riqueza, alto valor añadido e inductor de la cooperación y el comercio internacional.

- Realiza una actividad muy relevante en I+D+i e intensiva en capital, actuando como motor de innovación de la economía, con una gran capacidad de generación de conocimiento y movilización de recursos en el ámbito de la innovación.

- Presenta capacidad de generación de empleo de alta cualificación lo que se refleja en una mayor estabilidad y calidad en el empleo.

En cuanto al número de empresas del sector aeronáutico en España, según la información disponible procedente del Directorio Central de Empresas (DIRCE) del INE (Instituto Nacional de Estadística). El número de empresas que desarrollan la actividad de construcción aeronáutica y su maquinaria (clasificación 30.3 del CNAE 2009) fue de 94 empresas en el ejercicio del 2017. Al retroceder una década y observar su evolución, se comprueba como el sector no ha sido ajeno a la crisis económica vivida en España. De hecho, su comportamiento coincide exactamente con el periodo que se ha estandarizado y generalizado desde diferentes entes públicos y privados que señalan que, la crisis se inició en 2008 y finalizó en 2016 . Pues esta tendencia fue la que mantuvo el número de empresas aeronáuticas en España. Pues su máximo lo tuvo en el 2009 con 101, momento a partir del cual comenzó a perder empresas marcando su mínimo en el 2011 con 75 (ver gráfico 1).

Otra peculiaridad de la industria aeronáutica es el tamaño medio de sus empresas, pues en el 2017 el $63 \%$ de las empresas tenían más de 20 trabajadores y, el intervalo mayor era el de 20 a 49 trabajadores que representaba el $23 \%$ del total. Por lo que agrupando los intervalos de empleados, el mayor lo representan las empresas que tienen de 6 a 49 trabajadores, pero destaca sobre otras actividades industrias que se mantengan en el tiempo un $15 \%$ de media de empresas con una plantilla de 200 a 999 empleados. Sucediendo algo similar con las empresas con más de 1.000 trabajadores que representan entre un 3 y un $5 \%$ del sector a lo largo del tiempo. También se puede deducir que el descenso y posterior aumento del número de empresas que tuvo España en la última década, fue bastante homogéneo con referencia al estrato de empleados.

En cuanto a la distribución territorial a escala de Comunidad Autónoma, según la información del Directorio Central de Empresas del INE, en la década (2008 a 2017), son tres las Comunidades Autónomas que cuentan con

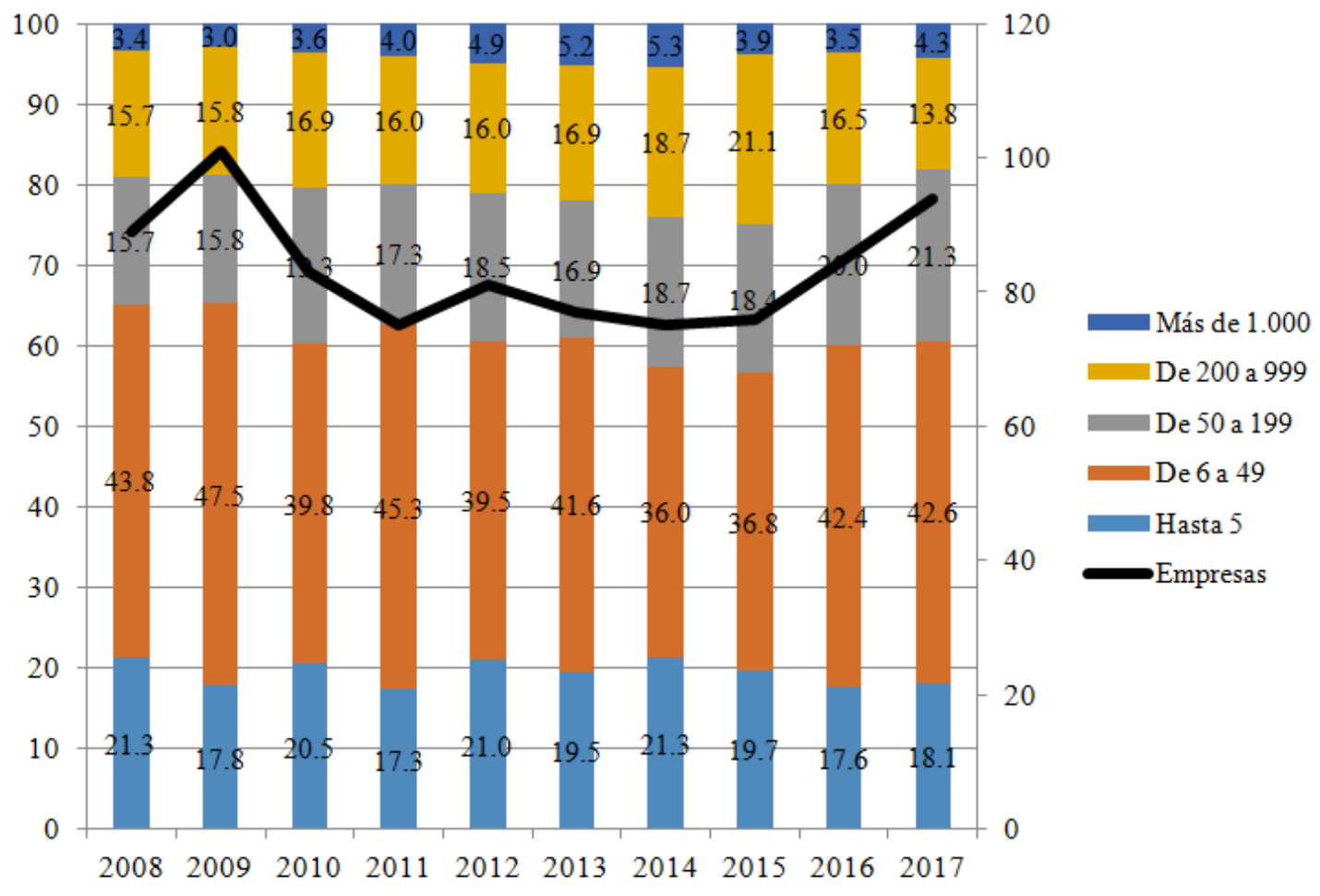

Gráfico 1 - Evolución del número de empresas del sector aeronáutico y porcentaje por estratos de empleo en España. 2008-2017. Fuente: elaboración propia a partir de datos del Instituto Nacional de Estadística, Directorio Central de Empresas, 2018. 
un mayor número de empresas del sector aeronáutico: Andalucía, Madrid y País Vasco. Concretamente en 2017 Andalucía con 25 empresas y, Madrid y el País Vasco con 21 respectivamente.

De hecho este trío autonómico siempre ha superado el $60 \%$ del total de las empresas del sector aeronáutico en España (ver gráfico 2). En el gráfico 2, sólo se representan las Comunidades Autónomas que han tenido alguna empresa en ese periodo de tiempo. Con lo que se desprende otra potencialidad de esta actividad industrial, que es comprobar cómo está presente en prácticamente todo el territorio español. Pues las únicas comunidades autónomas que no han tenido ni tienen empresas aeronáuticas son: Asturias, Cantabria, Navarra y las Islas Baleares. Además de Ceuta y Melilla. Mientras que existe un tercer grupo formado por las Comunidades Autónomas que tuvieron alguna empresa o la tienen en la actualidad, pero a lo largo del periodo 2008 al 2017 han dejado de tener representación como son: Murcia, Extremadura, Comunitat Valenciana y Canarias.

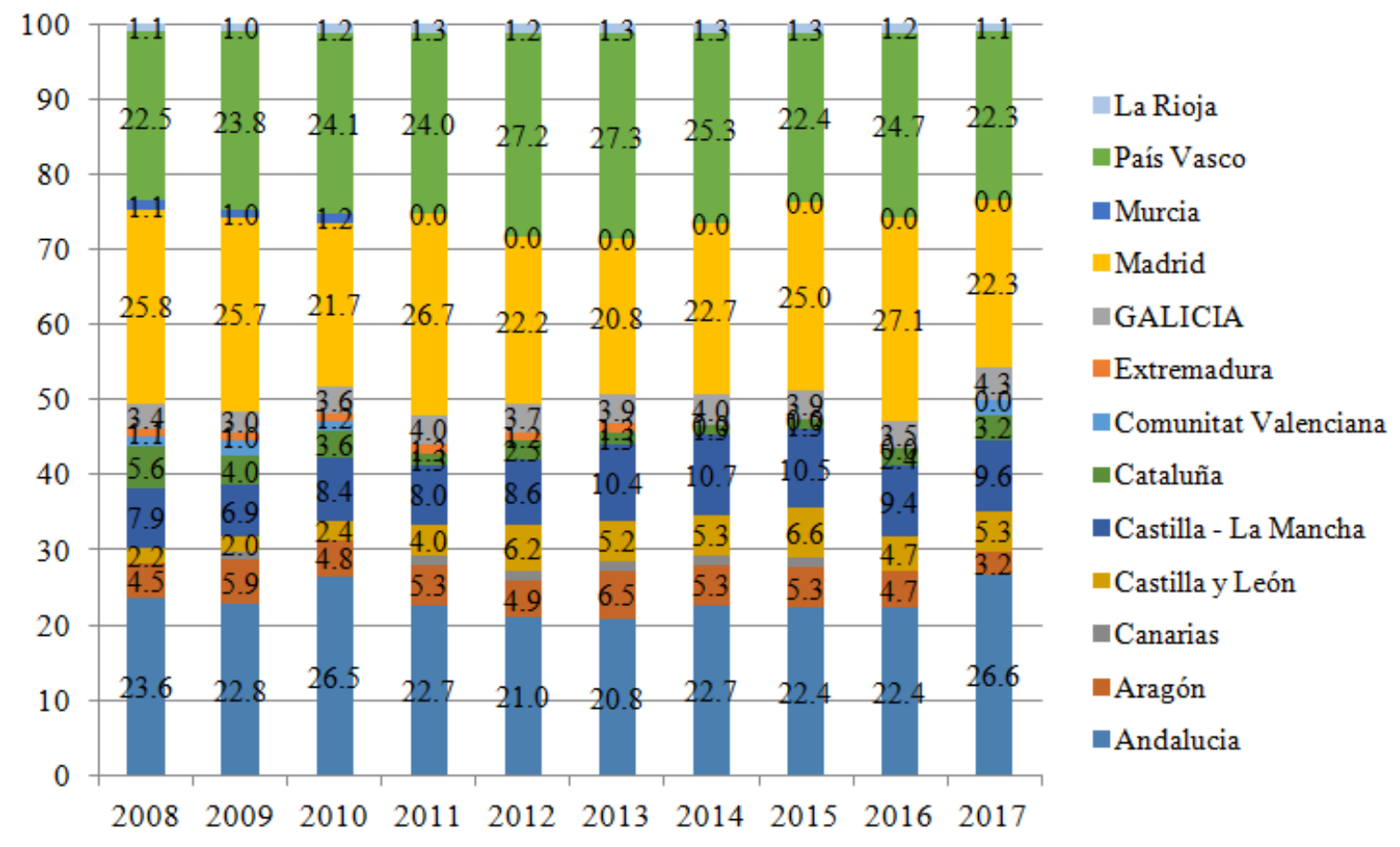

Gráfico 2 - Evolución del número y porcentaje de empresas del sector aeronáutico por Comunidad Autónoma en España. 20082017. Fuente: elaboración propia a partir de datos del Instituto Nacional de Estadística, Directorio Central de Empresas, 2018.

Los motivos de la representatividad del tridente que concentra la actividad aeronáutica española son variados. La importancia del sector aeroespacial en la región madrileña se explica por la existencia de un sistema integrado y diversificado de empresas, con una notable presencia de algunas actividades como estructuras de composites y otros materiales avanzados; comunicaciones, simulación y entrenamiento; sistemas de navegación y aviónica; mantenimiento aeronáutico y de helicópteros o ingeniería de propulsión. Dichas actividades se concentran en tres grandes localizaciones de la industria madrileña: Getafe-Parla-Móstoles, Tres Cantos y el Corredor de Henares.

En Andalucía la presencia del sector es creciente en los últimos años, debido, fundamentalmente, a la existencia de diversos grandes proyectos europeos que se están desarrollando en Andalucía, como son el programa del avión de transporte militar A-400M y la localización de su montaje y pruebas de vuelo en Sevilla. De hecho EADS (European Aeronautic Defence and Space) sólo tiene 3 centros de integración de aviones en Europa, localizados en Hamburgo, Toulouse y Sevilla. Por otro lado, la producción en la región se está diversificando, lo que le permite disminuir riesgos y aumentar las posibilidades de participación en otros programas. Aproximadamente, el 90\% de las actividades del sector se concentran en el eje Sevilla-Cádiz, destacando la presencia de Aerópolis (Parque Tecnológico Aeroespacial de Andalucía) en Sevilla y TecnoBahía en Cádiz.

En el País Vasco el sector aeroespacial está especializado en el diseño, desarrollo y fabricación de motores y componentes, dirigidos tanto a aeronaves como a actividades espaciales. Cuenta además con una infraestructura para realizar sus propios ensayos. El sector surgió a mediados de los años ochenta, fruto de la cooperación entre 
diferentes empresas que iniciaban su actividad en el sector. Entre las empresas se pueden destacar Industria de Turbo Propulsores (ITP), Aernnova o Sener (Ministerio de Energía, Turismo y Agenda Digital, 2011)

Una vez mostradas estas características básicas del sector aeronáutico en España en la última década, según los datos oficiales del INE. Se considera adecuado mostrar algunas de las peculiaridades que hemos conocido del sector después de haber analizado una serie de informes de organismos como TEDAE (Asociación Española de Empresas Tecnológicas de Defensa, Aeronáutica y Espacio). Por lo que primero utilizamos los datos oficiales, pero es información muy general y donde no se recogen todas las actividades incluidas dentro de la industria aeronáutica. Por lo que, como sucede con muchas otras actividades económicas, es necesario recurrir a fuentes de información más especializadas y, que nos permiten profundizar un poco más en la realidad territorial de esta industria.

Desde los informes más actuales realizados por TEDAE, se confirman ciertas magnitudes económicas con unas variables estadísticas que van más allá de las empresas del CNAE con código 30.3. Pues dentro del sector se incluyen otras actividades que son más "difíciles" de encuadrar como las que se dedican a la fabricación de equipos de telecomunicación para satélites, la fabricación de instrumentos de navegación, aparatos utilizados en aeronaves, la fabricación de sistemas de navegación aérea o la fabricación de dispositivos de iluminación para aeronaves.

La actividad del sector incluyendo la aeronáutica civil y militar casi alcanza los 8.000 millones de euros, el volumen de empleo supera los 100.000 empleados (40.000 directo e 60.000 indirecto). En cuanto a la facturación más del $11 \%$ se reinvierte en I+D+i y casi el $85 \%$ proviene de la exportación, dos aspectos muy característicos de la industria aeronáutica española. Al igual que comprobar que es 2,5 veces más productiva que la media española. De hecho, durante la década del 2005 al 2015, uno de los periodos centrales de la crisis socioeconómica vivida en España, la industria aeronáutica pasó de aportar al PIB generado por la industria un 2,2\% en 2005 a un 4,5\% en 2015. Presentando un crecimiento constante y continuo a lo largo de esos años, que se mantiene en la actualidad. Así como ver que por cada 100 euros invertidos por la industria aeronáutica en I+D+i se incrementa el PIB en 700 euros (TEDAE, 2015, 2017).

Otra peculiaridad del sector es que sus productos tienen un ciclo de vida muy largos y con unos costes de producción muy elevados. Con el hándicap añadido que los retornos de beneficios también son a largo plazo, aunque elevados.

Por otro lado nos encontramos con su alta vinculación y en ocasiones dependencia con la Administración, pues está vinculada a departamentos de investigación y educación, transporte, comercio, industria, defensa, seguridad o medio ambiente, que puede llegar a ocasionar graves problemas de coordinación si no son bien planteadas las políticas y convenios entre los gobiernos y la industria aeronáutica.

Por lo que el sector aeronáutico se caracteriza por necesitar empresas tractoras fuertes, personal altamente preparado y enormes recursos económicos. En compensación, los retornos que genera a la sociedad, en términos de reinversión, al Estado vía impuestos, el número de trabajos directos e indirectos, sus valores de comercialización exterior, etc., hacen de este sector uno de los más atractivos como receptor de inversiones de la Administración.

La crisis económica y la situación del mercado global actual, les afecta de forma desigual al subsector civil y al de defensa. La industria mundial de aeronáutica civil está dominada por la empresa norteamericana Boeing y la europea Airbus. Sin embargo, en los próximos años el dominio ejercido por estas dos empresas puede disminuir considerablemente, debido a la transformación de muchas empresas de escala regional de países como China y Rusia que han pasado a construir modelos de segmentos superiores y aptos para todos los mercados.

En España, el primer desarrollo del sector aeronáutico fue a mediados del siglo XX. Gracias a la iniciativa pública a través de la empresa Construcciones Aeronáutica, S.A. (CASA), hoy integrada en el Grupo Airbus. Un segundo periodo de crecimiento fue desde principios de los años noventa y desde el País Vasco, por la iniciativa privada de empresas como Aciturri, Aernnova e ITP, entre otras, ha dado un nuevo impulso al sector civil.

La naturaleza estratégica del sector aeronáutico hace que la participación y la implicación de los estados sea muy relevante, tanto a nivel de cliente, como de regulador, como de proveedor de fondos de I+D+i, (en forma de subvenciones para las actividades o como financiador de los programas de desarrollos más cercanos al mercado), o como accionista en diversos casos. Esto es muy importante e interesante para la geografía económica, pues la búsqueda de la constitución de esas redes entre diferentes agentes y características del territorio es algo que no se les escapa a los responsables de estas empresas. Quizás de la mano de la influencia que siempre han tenido de la Administración pero también ante la necesidad de tener que recurrir a otras empresas para completar un círculo de producción tan complejo como es el de la industria aeronáutica.

Un ejemplo claro y actual es la estrategia de especialización inteligente (RIS3) que significa que las estrategias de innovación nacionales o regionales tienen que definir prioridades para crear ventajas competitivas me- 
diante el desarrollo y la adecuación de los puntos fuertes de la investigación y la innovación a las necesidades empresariales, a fin de abordar las oportunidades emergentes y los avances del mercado de manera coherente y evitar, al mismo tiempo, la duplicación y fragmentación de los esfuerzos.

La Comisión propuso que estas estrategias fueran una condición previa para la financiación del FEDER. En consecuencia, los Estados miembros y regiones de la UE debían establecer las estrategias de RIS3 antes de que se aprobaran los programas operativos que respaldan estas inversiones.

En el caso de España, este trabajo concluyó en 2014, cuando se presentaron las RIS3 de todas las Comunidades Autónomas. Desde el gobierno de Galicia, se reiteró la significación que debe de tener en un futuro próximo la industria aeronáutica dentro de su estructura socioeconómica y las diferentes actuaciones realizadas para llegar a este objetivo.

\section{ANTECEDENTES Y SITUACIÓN ACTUAL DEL SECTOR EN GALICIA}

Dentro de España existen territorios que tienen un volumen de actividad relacionada con la industria aeronáutica mucho mayor que la presente en Galicia, como Madrid, Andalucía o el País Vasco. Sin embargo el caso gallego está adquiriendo cada vez mayor protagonismo debido a la implicación del gobierno autonómico, el crecimiento de las pocas pero grandes empresas localizadas en Galicia y, principalmente por la creación de un entramado, de una red, donde están presentes las empresas, la administración, la universidad y asociaciones que están creando unos cimiento sólidos de innovación y desarrollo sobre la industria aeronáutica.

La industria aeronáutica en Galicia cuenta con más de 40 empresas, ofrece trabajo directo e indirecto a más de 1.500 personas y tiene un volumen creciente de negocio de más de un 7\% en la producción directa (2016). Aunque no son valores muy importantes son varios los motivos que permiten pronosticar que la industria aeronáutica gallega es un sector "que tendrá futuro" en esta Comunidad Autónoma (CINAE, 2018).

Uno de los motivos, como veremos, es la predisposición de la Xunta de Galicia por involucrarse en su desarrollo y haber consolidado la colaboración con otros agentes como la Zona Franca de Vigo o la Universidad de Vigo para la potenciación y desarrollo de esta industria, a través de distintas políticas de apoyo.

Además desde la iniciativa privada, también se han comenzado a realizar actuaciones que tendrán un gran calado en el desarrollo a corto plazo del sector aeronáutico en Galicia. De hecho en el 2016, se realizó una inversión de más de 75 millones de euros en mejoras productivas para proveer (en diferentes niveles de subcontratación) a los cuatro mayores fabricantes de aviones del mundo: Airbus, Embraer, Boeing y Bombardier.

Una de las empresas es COASA, con sede en el Parque Tecnológico de Galicia en San Cibrao das Viñas (Ourense), que inyectó 45 millones de euros en sus instalaciones, para acondicionarlas a nuevos pedidos y llegar a nuevos mercados. Tras unas breves consultas informales, nos confirmaron que poseen convenios con pedidos de un gran país como la India que incrementará de forma importante la actividad de la planta y de sus principales auxiliares gallegas especializadas en mecanizado: Utingal (en Tui, Pontevedra) y Laddes Work, en Ourense. El principal cliente de COASA es Airbus, que al ser una de las empresas del sector más importantes del mundo le permite trabajar con "tranquilidad" e incrementar algunos riesgos. Algunos de sus productos son vendidos al $100 \%$ al mismo cliente.

Otra gran empresa gallega del sector es Delta Vigo, una fábrica de pura ingeniería en pleno proceso de expansión con capital cien por cien gallego. La empresa tiene plantas en Vigo e Illescas (Toledo), y acaba de invertir 30 millones en su tercera factoría en Galicia. La compañía viguesa prevé elevar la facturación desde los 22 millones actuales hasta los 60 , en el horizonte del 2020, con pedidos ya contratados para este periodo.

Otro proceso de expansión se promueve desde UDEGA, la Unión de Empresas Gallegas de Aeronáutica, con una inversión de 4 millones de euros para mejorar las instalaciones y desarrollar nuevas piezas de avión, de la que hablaremos en el próximo apartado.

En los últimos años la parte pública también se ha volcado en impulsar el sector aeronáutico gallego. Una muestra es la apuesta que el Instituto Nacional de Técnica Aeroespacial (INTA), organismo dependiente del Ministerio de Defensa, ha hecho por Galicia al fijar en Lugo la base del Centro de Investigación Aerotransportada de Rozas (CIAR), presupuestado en 10 millones de euros, a los que la Xunta prevé inyectar 45 más hasta el 2020. En la nómina de futuros socios tecnológicos figuran potencias como Airbus, Boeing Indra, Inaer o Thales. Otra actuación significativa fue la creación de la Fundación Centro de Innovación Aeroespacial de Galicia, impulsada por el Consorcio de la Zona Franca de Vigo y la Universidade de Vigo. 
En cuanto a los grandes datos económicos del sector en Galicia, aún no son muy representativos, pero hay que tener en cuenta dos factores: por un lado que es un sector que lleva poco tiempo en funcionamiento y, en segundo lugar que la calidad de la producción es de alto valor añadido pues proviene directamente de diversos procesos de ingeniería. Según los datos del TEDAE, Galicia representa un poco más del $4 \%$ del empleo y número de empresas de la industria aeronáutica de España. Sin embargo, ya existen estimaciones que se mantendrá a lo largo de los próximos años un incremento regional de más del 12\% en el empleo y del 10\% en la facturación.

De todos modos, al aspecto al que desde este trabajo se le quiere dar mayor importancia, es la presencia de una serie de agentes en este territorio del noroeste de la península lbérica que le dan un carácter innovador y una capacidad de crecimiento y expansión esperanzadores. A continuación se presentan brevemente, las características de cada uno de los agentes que forman esta red alrededor de la industria aeronáutica en Galicia (ver figura 1).

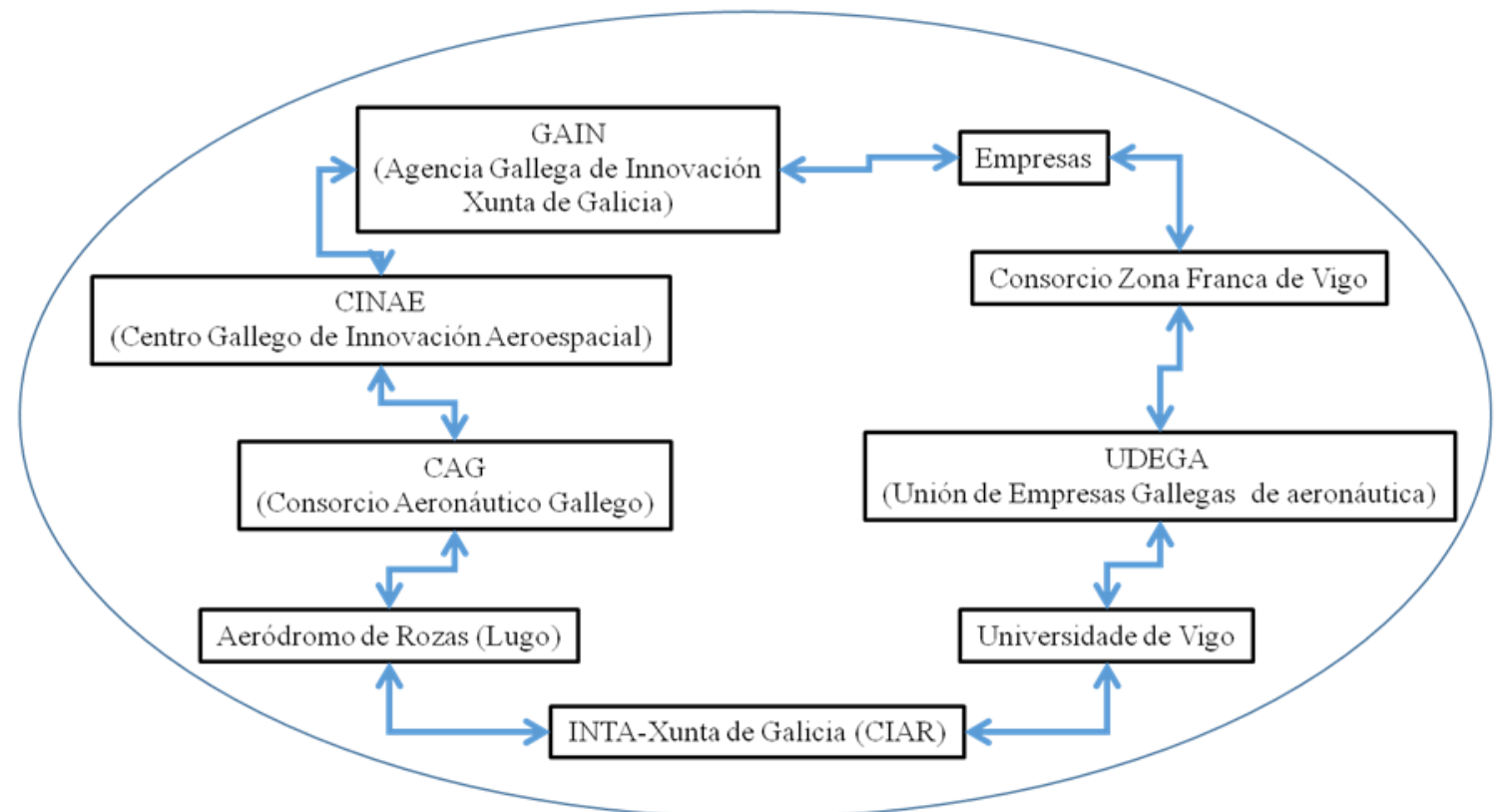

Figura 1 - Relación de agentes públicos, privados y empresariales vinculados con la industria aeronáutica en Galicia. Fuente: elaboración propia.

La Agencia Gallega de Innovación GAIN, es una agencia pública autonómica, adscrita a la Consellería de Economía, Emprego e Industria pero con personalidad jurídica propia, que tiene como finalidad fomentar y vertebrar las políticas de innovación en las administraciones públicas gallegas, y el apoyo e impulso del crecimiento y de la competitividad de las empresas gallegas, a través de la implementación de estrategias y programas de innovación eficientes. Aunque actúa sobre todo el territorio gallego y todas las actividades económicas, una de sus líneas prioritarias es la industria aeronáutica. La sede de la agencia está en la ciudad de Santiago de Compostela (GAIN, 2018).

El Centro gallego de Innovación Aeroespacial (CINAE) es una fundación privada sin ánimo de lucro fundada por la Universidad de Vigo, el Consorcio Zona Franca de Vigo, AIRBUS Defensa y Espacio, UDEGA, Delta Vigo y el Consorcio Aeronáutico Gallego. Sus orígenes se remontan a la actividad tecnológica del Grupo Aeroespacial de la Universidad de Vigo centrado en el desarrollo de pequeños satélites. Como centro se constituyó en el 2007 con el proyecto Xatcobeo y continuó con Humsat y otros proyectos en cooperación con el Instituto Nacional de Técnica Aeroespacial (INTA), la Agencia Espacial Brasileña (AEB), la Agencia Espacial Europea (ESA) y la Oficina de Naciones Unidas para Asuntos del Espacio Exterior (UNOOSA).

En la actualidad el centro se financia el $60 \%$ con fondos privados y un $40 \%$ con fondos públicos. El objetivo principal del CINAE es contribuir al fortalecimiento, potenciación, crecimiento y mejora de la competitividad en la industria de las tecnologías aeroespaciales de Galicia mediante el logro de la excelencia en la investigación y el desarrollo tecnológico. El equipo está compuesto por ingenieros de telecomunicaciones, ingenieros aeronáuticos con especialización en el sector aeroespacial, ingenieros industriales y físicos. La sede del centro está en Parque Empresarial Porto do Molle, en el municipio de Nigrán dentro del área de influencia de Vigo (CINAE, 2018). 
EI CAG (Consorcio Aeronáutico Gallego), también fue creado en el 2007 formado por 53 empresas pertenecientes al sector metalúrgico gallego, con el fin de promover y desarrollar el sector aeronáutico en Galicia y alcanzar un mayor nivel de innovación, investigación y competitividad, en todo el sector. El CAG, entre otras organizaciones en las que participa, es patrono fundador del CINAE y en actividades tan importantes como proyecto UAV'S Initiative de As Rozas (Lugo) o la creación del grado universitario de Ingeniería Aeroespacial de la Universidade de Vigo. EI CAG tiene su domicilio social en la ciudad de Vigo (CAG, 2018).

INTA - CIAR. La Xunta de Galicia está realizando una apuesta decidida por el desarrollo de un centro aeronáutico de referencia europea en Galicia. Alrededor del Parque Tecnológico de Rozas, aprovechando el aeródromo de Rozas (Castro de Rei, Lugo) y la creación del futuro Centro de Investigación INTA-Xunta (CIAR), dependiente del Instituto Nacional de Técnica Aeroespacial del Ministerio de Defensa, en el que se invertirá 55 millones de euros hasta 2020.

En el marco de esta política de promoción del centro tecnológico, el gobierno gallego lanzó la Civil UAVs Initiative para la utilización de sistemas aéreos no tripulados en el ámbito civil y en la mejora de la prestación de servicios públicos (Civil UAVs Initiative, 2018).

Enmarcada en la política de impulso del centro de investigación y tecnología aeronáutica, el gobierno gallego y el instituto Nacional de Técnica Aeroespacial (INTA) ya realizaron una inversión de 10 millones de euros en la adaptación de las infraestructuras del aeródromo de Rozas para acoger al Centro Mixto de Investigación INTAXunta (CIAR). Con el objetivo final de convertirse en el centro de ensayos para el desarrollo de aeronaves y la evaluación de campañas. Posteriormente, la Xunta de Galicia tiene en marcha un procedimiento de compra pública pre-comercial a nivel internacional de hasta 25 millones de euros, para la búsqueda de uno o varios socios tecnológicos industriales, especializados en sistemas aéreos no tripulados, que activen el desarrollo del centro tecnológico.

UDEGA S.L. (Unión de empresas gallegas de aeronáutica), se creó en 2012 de la unión de varias empresas gallegas, con el ánimo de unificar métodos de trabajo y su relación con el contexto externo para optimizar recursos materiales y humanos mediante la cooperación industrial. Capaces de abordar un amplio abanico de proyectos, UDEGA se constituye como un referente a escala española y presencia internacional. El nutrido parque de maquinaria unida a la amplia experiencia acumulada por todas las plantas en diversidad de sectores, como el naval, la automoción o el aeronáutico, hace de UDEGA una gran empresa, capaz de ofrecer a sus clientes variedad de productos y servicios relacionados con la industria aeronáutica (UDEGA, 2018).

El CZFV (Consorcio Zona Franca de Vigo) es una institución pública que gestiona una zona franca de comercio internacional y actúa como agencia de desarrollo económico. Tiene como objetivo principal fomentar el desarrollo económico del área de influencia de Vigo y Galicia en general. Desde el consorcio siempre muestran las ventajas que pueden tener los empresarios y emprendedores para instalar sus centros de negocio en este territorio. De hecho, CZFV posee la capacidad y conocimiento tanto para buscar las mejores opciones para los grandes y pequeños negocios, así como para encontrar el emplazamiento más adecuado para la empresa, dispone de las mejores instalaciones, capital, información, formación y conocimiento.

Al igual que con otras actividades económicas, mantiene una línea de actuación propia y exclusiva con la industria aeronáutica, participando en gran parte de los proyectos vinculados con el sector en Galicia (CZFV, 2018).

A lo largo de este trabajo, también se está comprobando la implicación de la Universidade de Vigo con el desarrollo de la industria aeronáutica en Galicia. Incluso se pude considerar como uno de los agentes que estuvo presente desde los inicios y primeras actuaciones de esta red innovadora, pues participó en la creación del CINAE o el CAG. Así como ser el único centro de Galicia en el que se puede obtener el grado de Ingeniería de Tecnologías de Telecomunicaciones y desde hace un par de años se imparte el grado de Ingeniería Aeroespacial, lo que asegura la creación de un cierto volumen de mano de obra altamente cualificada. La Universidade de Vigo tiene campus en las ciudades de Vigo, Ourense y Pontevedra.

\section{CONCLUSIONES}

Antes de desarrollar una breve reflexión final sobre el sector aeronáutico en Galicia. Consideramos que es muy importante dejar claro que nos encontramos ante un primer trabajo, una primera toma de contacto con un sector económico que en el territorio español y en concreto en Galicia, presenta un bagaje de actividad reciente. Sin embargo pensamos que muy importante y a tener en cuenta a corto y medio plazo. De hecho, se trata de esas 
actividades industriales que se catalogan como estratégicas a escala internacional y que presenta un mercado aun "por ocupar", en el que los territorios más desarrollados desde el punto de vista tecnológico están planteando diferentes estrategias para ocuparlo. De ahí el interés y esfuerzo de conocer este sector y más al ver que Galicia posee una red de innovación vinculada con esta actividad. Por lo que este estudio hay que interpretarlo con cautela, y como una base de análisis que utilizaremos en próximas investigaciones para seguir profundizando en las características de la industria aeronáutica.

De todos modos, una de las conclusiones a las que sí se llega en este trabajo es comprobar cómo en Galicia existe una red de innovación en la que están presentes todo tipo de agentes públicos, privados y un conjunto de empresas que parecen constituir unos cimientos muy adecuados para el desarrollo de este sector y que adquiera mayor protagonismo en la estructura socioeconómica de Galicia. Con lo que un segundo paso a efectuar en próximas investigaciones, será analizar el grado de relación que existe entre cada uno de los agentes.

Otra conclusión que se aprecia en el territorio gallego, es la existencia de una serie de líneas de apoyo promovidas desde la administración pública, muy especializada y dirigida con exclusividad hacia el sector aeronáutico, como son los casos de Civil UAVs Initiative o la dinamización del aeródromo de Rozas, entre otras. Muy superiores en número y dotación a la que encontramos en territorios próximos o con características similares a las que puede tener Galicia.

Esta ventaja se confirma al observar como desde todos los entes relacionados con el sector aeronáutico, se afirma que para su existencia se requiere de un decidido y permanente apoyo de las instituciones públicas. Así como, que el sector aeronáutico español debe aspirar a seguir manteniendo su presencia en los proyectos y productos de vanguardia y de última generación, frente a la competencia de las empresas de los nuevos países que se incorporan al sector.

De todos modos, en España y el caso concreto de Galicia el comportamiento de todas estas asociaciones, la creación de infraestructuras como el aeródromo de Rozas o los centros tecnológicos, nos despiertan dudas y es una incógnita, por lo que tendremos que estar atentos a su evolución y ver cómo se comporta dentro de un sector altamente competitivo y tecnológico.

Por último, señalar que se aprecia una clara especialización y distribución territorial del sector en el sur de Galicia, principalmente en el área de influencia de las ciudades de Vigo y Ourense. No sorprende porque se tratan de territorios donde otros sectores económicos tienen mucha significación a escala internacional como la automoción, textil-moda o agroalimentario. Sin embargo, si es menos normal no encontrar mayor implicación del área de influencia de A Coruña o Santiago de Compostela, más allá de la presencia de alguna empresa o las gestiones realizadas desde la propia sede de la Xunta de Galicia.

\section{BIBLIOGRAFIA}

AGENCIA GALLEGA DE INNOVACIÓN (GAIN) (2018) http://gain.xunta.gal

ALONSO, J. L.; APARICIO, L. J. y SÁNCHEZ, J. L. (2004). Recursos territoriales y geografía de la innovación industrial en España. Ediciones Universidad de Salamanca. Salamanca. Páginas: 352.

ALONSO, Ma . P. y MIRAMONTES, Á. (2003). "La significación de un medio innovador local dentro de la industria de muebles de Galicia: A Estrada (Pontevedra)". Boletín de la Asociación de Geógrafos Españoles. Número 36. Asociación de Geógrafos Españoles. Madrid. Páginas: 93-102.

AYDALOT, P. (1986). Milieux innovateurs en Europe. GREMI. Paris.

BORRÁS, S. Y EDQUIST, C. (2013): "The choice of innovation policy instruments", Technological Forecasting and Social Change, 80, 1513-1522.

CAMAGNI, R (1991). Innovation networks. Spatial perspectives. GREMI-Belhaven Press, Londres.

CAMAGNI, R. (2003). "Incertidumbre, capital social y desarrollo local: enseñanzas para una gobernabilidad sostenible del territorio". Investigaciones Regionales. Asociación española de ciencia regional. Mundi-Prensa. Libros. Número: 2. Madrid. Páginas: 31-58.

CARAVACA BARROSO, I.; GONZÁLEZ, G.; MÉNDEZ, R. y SILVA, R. (2002). Innovación y territorio: análisis comparado de sistemas productivos locales en Andalucía. Junta de Andalucía. Consejería de Economía y Hacienda. Sevilla. Páginas: 392.

CENTRO GALLEGO DE INNOVACIÓN AEROESPACIAL (CINAE) (2018) https://www.cinae.org/

CIVIL UAVS INITIATIVE (2018) http://www.civiluavsinitiative.com/

CONSORCIO AERONÁUTICO GALLEGO (CAG) (2018) http://www.consorcioaeronautico.com/ 
CONSORCIO ZONA FRANCA DE VIGO (CZFV) (2018) https://www.zfv.es/portal/index.html INSTITUTO NACIONAL DE ESTADÍSTICA (INE) (2018) http://www.ine.es/

MAILLAT, D. (1995). "Les milieux innovateurs”, Sciencies Humaines, 8. Páginas: 41-42.

MÉNDEZ GUTIERREZ DEL VALLE, R. (2000). "Procesos de innovación en el territorio: los medios innovadores". Innovación, pequeña empresa y desarrollo local en España. Editorial Civitas. Madrid. Páginas: 23-59.

MÉNDEZ, R. y ALONSO, J. L. (coords.) (2000). Innovación, pequeña empresa y desarrollo local en España. Civitas. Madrid. Páginas: 323.

MÉNDEZ, R. y ALONSO, J. L. (eds.) (2002). Sistemas Locales de Empresas y redes de innovación en Castilla - La Mancha y Castilla y León. Universidad de Salamanca.

MÉNDEZ GUTIERREZ DEL VALLE, R. (2006). "La construcción de redes locales y los procesos de innovación como estrategias de desarrollo rural”. Problemas del Desarrollo. Revista Latinoamericana de Economía, vol. 37, núm. 147. Universidad Nacional Autónoma de México. México. Páginas: 217-240.

MIRAMONTES, A. Y ALONSO, Ma. P. (2015) "Sistemas productivos locales en la industria del mueble en Galicia (España). Su situación ante la crisis". Anales de Geografia de la Universidad Complutense de Madrid, volumen 35. Editorial Servicio de Publicaciones de la Universidad Complutense de Madrid, Madrid (España). Páginas: 22 inicial 63 final 94

MINISTERIO DE ENERGÍA, TURISMO Y AGENDA DIGITAL (2011) El sector aeronáutico y espacial español: Situación actual y perspectivas, MCA-UGT (Metal, Construcción y Afines de la Unión General de Trabajadores, Federación de Industria) Madrid, $122 \mathrm{p}$.

SALOM CARRASCO, J. (2003). "Innovación y actores locales en los nuevos espacios económicos: un estado de la cuestión". Boletín de la Asociación de Geógrafos Españoles. Número: 36. Madrid. Páginas: 7-30.

SALOM, J. y ALBERTOS, J.M. (2009). Redes socioinstitucionales, estrategias de innovación y desarrollo territorial en España. Colección Desarrollo Territorial. Serie Estudios y Documentos, 6. Publicaciones de la Universitat de Valencia. Valencia. Páginas: 202.

SALOM, J. y ALBERTOS, J. M., 2013). "Los efectos de la crisis económica en los distritos industriales valencianos: ¿hacia nuevos desequilibrios territoriales?", En Crisis económica e impactos territoriales. Actas de las V Jornadas de Geografía Económica AGE Universitat de Girona. Páginas 267-284.

SFORZI, F. (1999). "La teoría marshaliana para explicar el desarrollo local", en Rodríguez Gutierrez, F., (coord.), Manual de desarrollo local, Trea ediciones, Gijón. Páginas: 13-22.

SMITS, R, KUHLMANN, S. and SHAPIIRA, PH (2010): The Theory and Practice of Innovation Policy.

An International Research Handbook.Cheltenham, UK, Edward Elgar, $496 \mathrm{pp}$.

TEDAE (2015). Retos del Sector Aeronáutico en España. Guía Estratégica 2015-2025, Madrid, 27 p (https://www.tedae.org/es)

TEDAE (2017). La Aeronáutica española, una apuesta de futuro, Madrid, 2 p. (https://www.tedae.org/es)

UNIÓN DE EMPRESAS GALLEGAS DE AERONÁUTICA (UDEGA) (2018) http://udega.com/es/

VÁZQUEZ BARQUERO, A. (1999). Desarrollo, redes e innovación. Lecciones sobre desarrollo endógeno. Pirámide. Madrid. Páginas: 268. 